\title{
Opening Multiwall Carbon Nanotubes in a Transmission Electron Microscope
}

\author{
H. Ye*, N. Naguib*, Y. Gogotsi*, A. G. Yazicioglu**, and C. M. Megaridis** \\ * A. J. Drexel Nanotechnology Institute and Department of Materials Engineering, Drexel \\ University, Philadelphia, Pennsylvania 19104 \\ ** Department of Mechanical Engineering, University of Illinois at Chicago, Chicago, Illinois \\ 60607
}

The opening and filling of carbon nanotubes (CNTs) is of great importance for many applications, such as electrochemical energy or gas storage systems, biological nanosensors, and nanofludic devices. While filling CNTs can be accomplished by directly sucking liquid or molten materials with capillary force ${ }^{[1]}$, it is not easy to open the CNTs in a controlled way. Various methods have been developed to open multiwall carbon nanotubes (MWNTs), including chemical treatments with nitric acid or oxidants ${ }^{[2]}$, thermal treatment by heating in an oxidizing atmosphere ${ }^{[3]}$ and abrasive treatment by rubbing the MWNTs on diamond disks ${ }^{[4]}$. However, in all these methods the opening only happens at the tips of the MWNTs, and uncontrolled damages to tube walls might occur. There is also no control over the location of the opening on the tube surface. Here we report a new method for opening the side wall of MWNTs by using converged electron beams in a transmission electron microscope (TEM). With this method we can specifically select the positions on nanotubes where we would like to perform the puncture. This is of importance for nanofluidic devices and other applications in which nanotube machining must be done at nanoscale.

The MWNTs investigated were hydrothermally synthesized as described in Ref. ${ }^{[5]}$. The produced MWNTs contained entrapped liquid whose main component was water. A high resolution TEM (JEOL 2010F) was used to open the MWNTs. Fig. 1 shows a typical TEM image of the hydrothermal MWNTs with water inclusion. When the electron beam in TEM is converged rapidly (within 1 second) and precisely on the region of the water inclusion, a puncture of the nanotube can be immediately observed, as shown in Fig. 2. The hole formed after puncture typically has an elliptic shape that crosses the nanotube with a larger size than the inner tube diameter. Depending on the irradiation conditions, the holes can be produced on both the front and back sides of the nanotube. More efficient and precise control of the opening effect can be realized when the electron probe in scanning transmission electron microscopy (STEM) mode is used for irradiation.

The strong interaction between water inclusion and carbon tube walls under electron irradiation may account for the phenomenon of an opening in the MWNTs. It is known that water heated to high temperature by a convergent electron beam can react with carbon by the reaction: $\mathrm{C}+\mathrm{H}_{2} \mathrm{O} \rightarrow \mathrm{CO}+$ $\mathrm{H}_{2}$, dissolving the tube walls and hydrating the graphene sleeve edges ${ }^{[6]}$. At the initial stage of the reaction, part of the graphite layer can be detached from the inner tube walls and bent toward the liquid inclusion, as shown in Fig. 3. This demonstrates the strong interaction between the tube wall and liquid inclusion. Upon intense electron beam heating, the reaction would finally result in the local dissolution of the tube walls, forming the hole as observed in Fig. 2. Since the position of the puncture can be specifically selected by moving the electron probe, precise tube nano-machining for nanofluidic devices is feasible. 


\section{References}

[1] E. Dujardin et al., Science 265 (1994) 1850.

[2] S.C. Tsang et al., Nature 372 (1994) 159.

[3] S.C. Tsang et al., Nature 362 (1993) 520.

[4] G. Maurin et al., Carbon, 39 (2001) 1273.

[5] Y. Gogotsi et al., J. Mater. Res., 15 (2000) 2591.

[6] Y. Gogotsi et al., Chem. Phys. Lett., 365 (2002) 354.

[7] This research was supported by the US National Science Foundation under NIRT grant CTS0210579. The microscope used was operated at LRSM, University of Pennsylvania.

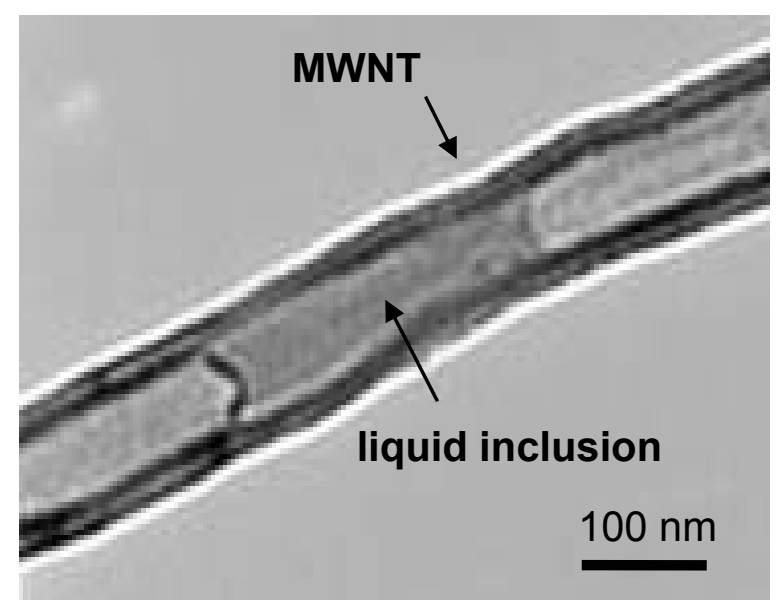

FIG. 1. A typical hydrothermally produced MWNT with a liquid inclusion.

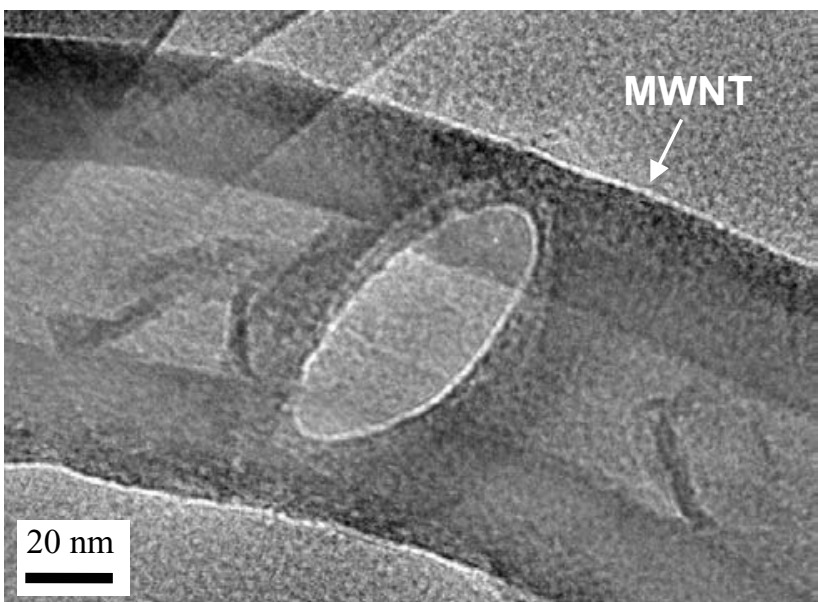

FIG. 2. An elliptic hole in the tube wall formed by conducting a converged electron beam on the liquid inclusion of the hydrothermal MWNT.

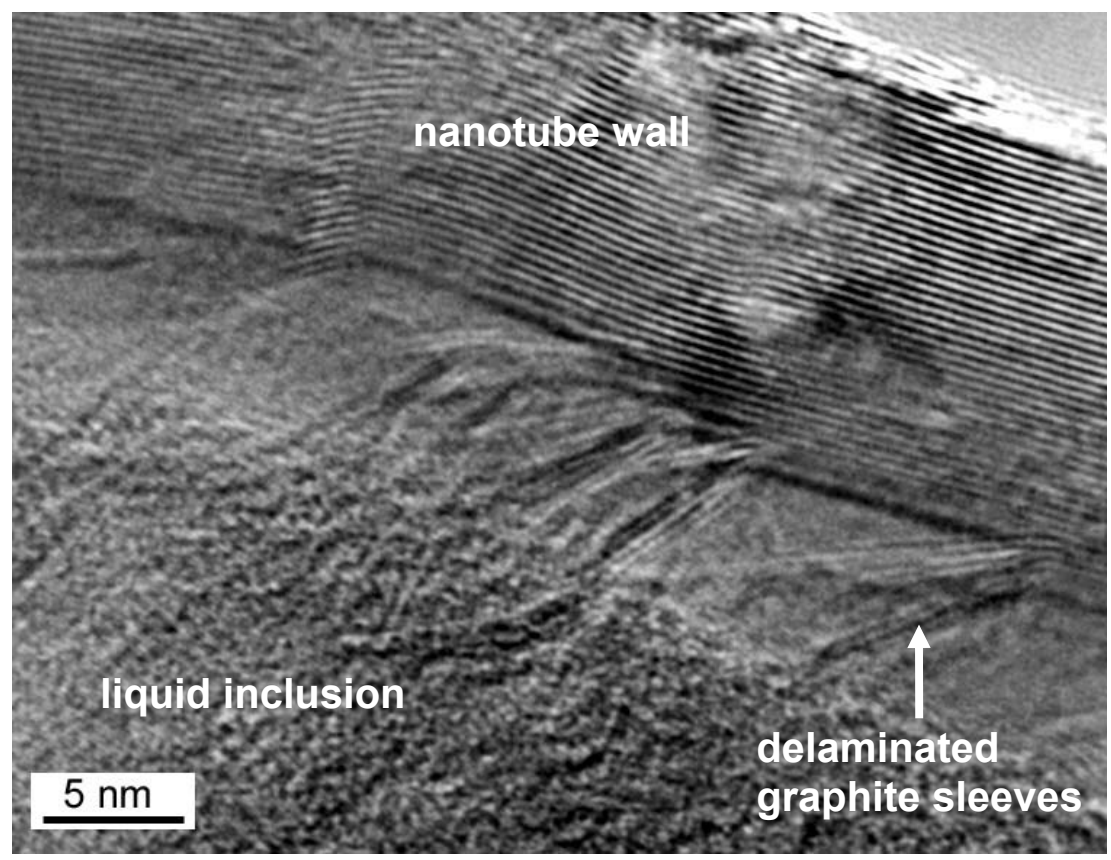

FIG. 3. High resolution TEM image of the tube wall, showing that the strong interaction between liquid and the carbon tube wall delaminates the graphite sleeves and bends them toward the liquid inclusion. 